\title{
VALIDACIÓN TRANSCULTURAL DE INTERVENCIONES CLÍNICAS BASADAS EN LA EVIDENCIA PARA EL TRATAMIENTO DE LOS TRASTORNOS DE ANSIEDAD
}

\author{
Georgina Cárdenas ${ }^{1}$, Cristina Botella ${ }^{2,4}$, Rosa M. Baños ${ }^{3,4}$, Soledad Quero ${ }^{2,4}$, \\ Ximena Durán ${ }^{5}$ y Anabel de la Rosa ${ }^{6}$ \\ ${ }^{1}$ Universidad Nacional Autónoma de México, México \\ ${ }^{2}$ Universidad Jaime I, Castellón, España \\ ${ }^{3}$ Universidad de Valencia, Valencia, España \\ ${ }^{4}$ CIBER de Fisiopatología de la Obesidad y Nutrición (CIBEROBN) \\ ${ }^{5}$ Soluciones Virtuales para la Salud, México \\ ${ }^{6}$ Facultad de Estudios Superiores Iztacala, Universidad Nacional Autónoma de México, México
}

\begin{abstract}
Resumen: Las aplicaciones de tecnologías avanzadas han generado la emergencia de un espacio de investigación en ciencias del comportamiento. El creciente acervo de publicaciones, sobre la eficacia y eficiencia de programas de intervención para una serie de trastornos psicológicos, plantea la necesidad de fortalecerlos, evaluando su generalización a otros contextos culturales para contribuir a la medición de la validez externa. Mediante la colaboración de dos grupos de investigación, se realizó la validación transcultural de tres intervenciones empleando realidad virtual para el tratamiento de miedo a volar, agorafobia y miedo a hablar en público con un programa auto-aplicado, que demostraron previamente eficacia con población española. Se informan los resultados de eficacia y eficiencia de las intervenciones en población mexicana. Se discute la relevancia de la validez externa de los programas de tratamiento con incorporación tecnológica, que pueden contribuir a optimizar tratamientos y sistemas de valoración psicológica beneficiando los servicios de salud mental.
\end{abstract}

Palabras clave: Práctica basada en evidencia; tratamientos realidad virtual; programas auto-aplicados; validez externa; trastornos de ansiedad.

\section{Cross-cultural validation of evidence-based clinical interventions for the treatment of anxiety disorders}

\begin{abstract}
In recent years, applications of advanced technologies have led to the emergence of a research line in the behavioral sciences. The growing body of publications that report the efficiency and effectiveness of intervention programs that address a range of psychological disorders raises the need to strengthen them, assessing their generalizability to other cultures in order to assess the external validity. Through the collaboration of two research groups, a cross-cultural validation was carried out in Mexico, assessing two treatment programs using virtual reality to treat fear of flying, agoraphobia and fear of public speaking with a self-applied program, which previously demonstrated efficacy in Spain. In this paper we report the results of effectiveness and efficiency in the Mexican population. It is discussed the relevance to assess external validity of treatment programs incorporating technology, that might contribute to optimize treatment and psychological evaluation systems that will benefit mental health services.
\end{abstract}

Keywords: Evidence-based practice; virtual reality treatments; self-applied programs; external validity; anxiety disorders.

Recibido: 8 junio 2014; aceptado 26 junio 2014.

Correspondencia: Dra. Georgina Cárdenas-López, Universidad Nacional Autónoma de México, Facultad de Psicología. Tajin no. 14, Depto. 204, Colonia Piedad Narvarte, Delegación Benito Juárez, México D.F., C.P. 03000.
Correo-e:mgcl@unam.mx; www.ciberpsicologia.psicol. unam.mx 


\section{INTRODUCCIÓN}

Recientemente ha surgido un interesante y creciente acervo de literatura especializada, que informa acerca del empleo de tecnologías avanzadas en el área de la salud, revelando los continuos avances y descubrimientos para ayudar a pacientes con desórdenes psicológicos y para promover comportamientos positivos que conduzcan a una mayor calidad de vida. En nuestros días estas innovaciones ya no son vistas como ciencia ficción empleada por investigadores de realidad virtual o programadores inquietos.

El potencial de las aplicaciones de tecnologías avanzadas en el campo de la salud ha generado la emergencia de un espacio de investigación y desarrollo tecnológico en las ciencias del comportamiento. En los últimos años, los hallazgos y avances realizados por diversos investigadores, han contribuido a la generación de conocimiento para incorporar tecnologías de punta en este campo. La incorporación de las tecnologías de la comunicación e informática en el área de la salud mental y rehabilitación, es una realidad, en particular sus aplicaciones a la psicología clínica.

En particular, la existente capacidad de la tecnología de realidad virtual (RV) para crear, ambientes interactivos en 3D con estímulos que permiten que el comportamiento humano pueda ser motivado, así como también medido, ofrece opciones de evaluación y de intervención clínica que no eran posibles utilizando enfoques previamente disponibles. Desde hace un par de décadas un grupo de activos investigadores y clínicos que no sólo han reconocido el impacto potencial de la tecnología VR, sino que han generado una literatura de investigación importante donde VR puede aportar un valor añadido respecto a los métodos de evaluación e intervención tradicionales (Rizzo, Lange y Koenig, 2014).

Con base en estos informes, existe un consenso creciente de que la RV ahora se ha convertido en una herramienta prometedora en muchos ámbitos de la atención clínica y la investigación. Actualmente se está demostrando la eficacia de un número de áreas de la psicología clínica, neuropsicología y en la rehabilitación cognitiva y motora. La publicación de sus resultados, informa de a exitosa aplicación de la $\mathrm{RV}$, por ejemplo para la reducción del miedo en personas con fobias simples (Parsons y Rizzo, 2008; Powers y Emmelkamp, 2008), el tratamiento de trastorno de estrés postraumático (Cárdenas y De la Rosa, 2012; Cárdenas, De la Rosa, Flores y Durán, 2013; Cárdenas, De la Rosa, Durán, y Bouchard, 2014; Difede et al., 2007; López Soler, Castro, Alcántara y Botella, 2011; Rizzo, 2010; Rizzo et al., 2013; Rizzo, Difede, Rothnaum, y Reger, 2010; Rizzo et al., 2011; Rothbaum, Hodges, Ready, Graap, y Alarcón, 2001), alteraciones de la imagen corporal en pacientes con trastornos de la alimentación (Riva, 2011), la rehabilitación funcional de por ejemplo: derrame cerebral, lesión cerebral traumática, parálisis cerebral (Holden, 2005; Merians, Fluet, Qiu, Saleh, Lafond, y Adamovich, 2010).

Es importante mencionar que "ya existe una larga lista de intervenciones basadas en la práctica basada en la evidencia (PBE); aunque, claro está, dicha lista no es, ni mucho menos, definitiva y el trabajo de investigación análisis y recopilación no ha hecho más que empezar" (Botella, 2011, p. 21). Con el propósito de contribuir a esta tarea es porque se han iniciado esfuerzos importantes para evaluar la validez externa de los programas de intervención que se documentan como eficaces y promisorios para atender una serie de trastornos psicológicos debido a su eficacia comprobada y muchos otros se están evaluando con el propósito de refinarlos y fortalecerlos.

Sin embargo a la fecha, todavía se discute el uso de la investigación como guía para la prestación de los servicios clínicos. Por una parte, el escepticismo implícito en PBE sobre la idea de que la experiencia clínica del practicante es una base insuficiente para la práctica efectiva de su disciplina. Sin embargo, ese escepticismo que se manifestaba durante la década de los ochentas, no implicaba que la experiencia práctica y los conocimientos fueran irrelevantes e innecesarios, sino que ellos por sí solos, no resultaban suficientes para determinar la eficacia de una intervención psicológica. Afortunadamente, la definición revisada de PBE ahora prominente en la literatura médica profesional (Moriana y Martínez, 2011; Sacke- 
tt, Straus, Richardson, Rosenberg, y Haynes, 2000), así como en la literatura de servicios humanos incorporan el juicio profesional y los valores, así como las preferencias del cliente (Rubin, 2008).

Botella (2011) señala la importancia que tiene al evaluar el distinguir entre la eficacia y la efectividad o utilidad clínica, prestar atención a ambos aspectos. El eje de la eficacia, o de la validez interna, supone analizar rigurosamente la evidencia científica disponible, con la meta de valorar la efectividad de cualquier intervención dada que tiene validez interna en la medida que los arreglos en su diseño permitan determinar lógicamente que los resultados observados reflejan efectividad o falta de efectividad; frente a explicaciones alternativas.

El eje de la efectividad supone la evaluación de la generalización o validez externa de la administración de la intervención en varios contextos; la viabilidad de la intervención a través de pacientes y contextos, así como los costes y beneficios asociados con la administración de la intervención (Botella, 2011). En este sentido la validez externa de un estudio hace referencia a sí los resultados del estudio pueden o no ser generalizados y si los procedimientos de intervención, así como los resultados son aplicables al contenido social y cultural del cliente.

En México, la Encuesta Nacional de Epidemiología Psiquiátrica (2003) informó que los trastornos más frecuentes en la población son los trastornos de ansiedad (14.3\%), particularmente la fobia específica (FE; 7.1\%), seguida de fobia social (FS; 4.7\%) y el trastorno por estrés postraumático (TEPT) (1\%) (Medina-Mora, 2003). Dentro de los trastornos de ansiedad, el trastorno de agorafobia es un problema de salud mental grave e incapacitante a nivel mundial. La agorafobia es un temor irracional a estar en lugares públicos y aglomerados, y es considerado como la fobia más común y estresante en pacientes adultos. Los síntomas agorafóbicos son la aparición de ansiedad al encontrarse en lugares o situaciones donde sea difícil escapar, o donde, en caso de aparecer una crisis de pánico o síntomas similares, puede no disponer de ayuda. Los temores agorafóbicos se relacionan con situaciones como: permane- cer solo fuera de casa, estar en aglomeraciones, hacer filas largas, o viajar en transporte público. Estas situaciones se evitan, debido a la presencia de altos niveles de malestar y ansiedad o recurren a la compañía de un conocido para soportarlas (DSM-IV-TR).

Otro de los trastornos de ansiedad prevalentes en la población mexicana, es la fobia a volar (FV) una fobia específica del tipo situacional (DSM-IV-TR), que se define como un miedo intenso e irracional acerca de situaciones relacionadas a volar. Muchos individuos que padecen esta fobia informan preocupación y miedo desde el momento que saben que tienen que volar. Esto significa, días, semanas e incluso meses antes de volar. Este fenómeno se conoce como ansiedad anticipatoria. En algunas ocasiones, no es necesario que la persona vuele para sentir ansiedad, es decir, el hecho de llevar a alguien al aeropuerto, escuchar los motores del avión, ver un avión volar, o incluso ver una película relacionada con aviones provoca estrés intenso y sufrimiento. Las situaciones que los individuos con miedo a volar evitan pueden ser bastantes y no solo relacionarse con el hecho de estar sentado en el avión y volar sino también comprar boletos, ir al aeropuerto, empacar, ver películas o documentales acerca de aviones o de vuelos, etc. (Quero et al., 2012).

Dentro de la fobia social (FS) el miedo a hablar en público es otro de los trastornos de ansiedad, que ha sido reconocido en los últimos años, como un problema de salud mental, que sin embargo no es diagnosticada y atendida con oportunidad (Scheneier, Johnson, Horning, Liebowitz, y Weissman, 1992). La FS se caracteriza por la presencia de miedo intenso y persistente relacionado con una o varias situaciones en las que la persona puede actuar de modo ridículo o humillante delante de otros; es decir, se trata de situaciones en las que el individuo se expone a la observación o evaluación de otras personas (Botella, Baños, y Perpiñá, 2003).

Debido a que existe una alta prevalencia de los trastornos, y considerando la importancia de evaluar el eje de la efectividad, o utilidad clínica de programas con el empleo de RV, ya que en nuestro país, el desarrollo de este tipo 
de sistemas es incipiente, se puso en marcha un esfuerzo colaborativo entre los grupos de investigación de la Universidad Jaime I de Castellón (España) y la Universidad Nacional Autónoma de México (UNAM) que dio lugar a la instrumentación del proyecto apoyado por el Consejo Nacional de Tecnología de México, titulado "Desarrollo y evaluación de sistemas basados en tecnologías de realidad virtual para el tratamiento de trastornos de ansiedad" cuyo propósito fundamental consistió en llevar a cabo la validación transcultural y la evaluación de la eficacia y eficiencia de tres intervenciones para el tratamiento de miedo a volar, miedo a hablar en público y agorafobia, que demostraron eficacia con población española ya otros contextos sociales y culturales, específicamente con población mexicana. Para su instrumentación se realizaron ajustes a los ambientes de $\mathrm{RV}$, en los cuales los pacientes fueron expuestos en inmersión, considerando el contexto social y cultural de la población mexicana; favoreciendo en primer lugar un encuadre cultural; y segundo la posibilidad de que el usuario tuviera una mayor interacción con el sistema y finalmente una mejor ejecución práctica dentro de los mismos.

Una vez realizada la transferencia tecnológica de los sistemas tecnológicos, el equipo mexicano recibió entrenamiento del grupo de investigación español sobre la conducción de los protocolos de tratamiento, con el doble objetivo de: (a) ajustar la pertinencia cultural de los materiales de tratamiento y evaluación para población mexicana y b) replicar en la clínica de servicios psicológicos de la UNAM, los estudios llevados a cabo en España con resultados promisorios. Es así que el presente trabajo informa los resultados de eficacia y efectividad de tres estudios (Cárdenas et al., 2014; Cárdenas, Botella, Quero, de la Rosa, y Baños, 2014; Cárdenas et al., 2013); así como informa sobre la validez externa de los programas de intervención con población mexicana.

Por todo lo anterior, mediante el presente estudio queremos aportar evidencia sobre la validación transcultural de los programas de tratamiento con el objetivo de: (a) valorar la eficacia diferencial y la validación externa de tres programas de intervención españoles apoyados con tecnología para agorafobia, miedo a volar y miedo a hablar en público, adaptados para la población mexicana, y (b) analizar los efectos de los programas de intervención sobre las medidas clínicas de ansiedad fóbica, evitación, creencia sobre el pensamientos negativos; sintomatología asociada: depresión y ansiedad-estado; presencia y juicio de realidad; y satisfacción del tratamiento.

\section{MÉTODO}

\section{Participantes}

(1) Agorafobia. La muestra estuvo compuesta por 9 participantes que asistieron al Centro de Servicios Psicológicos Dr. Guillermo Dávila de la Facultad de Psicología de la UNAM para tratar el trastorno de Agorafobia. Cinco mujeres $(55.6 \%)$ y cuatro hombres $(44.4 \%)$. Todos los participantes cubrieron con el criterio del DSM-IV-TR para agorafobia. La edad media fue de 35.56 años $(D T=10.37$ años $)$ con un rango de 19 a 50 años. De entre los participantes todos contaban con estudios a nivel licenciatura.

(2) Miedo a volar en avión. La muestra se conformó con 9 participantes que asistieron al Centro de Servicios Psicológicos Dr. Guillermo Dávila de la Facultad de Psicología de la UNAM, para superar su miedo a volar. Ocho participantes eran mujeres $(88.9 \%)$ y un participante hombre $(11.1 \%)$; todos los participantes cumplían los criterios del DSM-IV-TR para fobia específica situacional (miedo a volar). La edad media fue de 42.22 años $(D T=15.18$ años) en un rango de 20 a 63 años. Siete participantes contaban con estudios a nivel licenciatura $(77.8 \%)$ y 2 con estudios de bachillerato $(22.2 \%)$; ninguno de ellos se encontraba tomando medicamento para el tratamiento del miedo a volar en avión.

(3) Miedo a hablar en público. La muestra de miedo a hablar en público estuvo conformada por 9 participantes que asistieron al Centro de Servicios Psicológicos Dr. Guillermo Dávila de la Facultad de Psicología de la UNAM, para recibir el programa de tratamiento para 
miedo a hablar en público. Cuatro participantes eran mujeres $(44.4 \%)$ y cinco hombres (55.6\%); todos los participantes cumplían los criterios del DSM-IV-TR para fobia social (miedo a hablar en público). La edad media era de 40.56 años $(D T=15.65$ años $)$ en un rango de 22 a 60 años. De entre los participantes 5 contaban con estudios a nivel licenciatura $(55.6 \%)$ y 4 con estudios de bachillerato $(44.4 \%)$.

\section{Instrumentos}

En este apartado se incluyen únicamente las medidas de autoinforme sobre variables transversales a los tres estudios: (a) medidas clínicas de ansiedad fóbica, evitación, creencia sobre el pensamientos negativos; (b) sintomatología asociada: depresión y ansiedad-estado; (c) variables de inmersión y juicio de realidad; y (d) satisfacción del tratamiento. Una descripción del protocolo completo puede consultarse en Cárdenas, Botella, Quero, de la Rosa et al., 2014, Cárdenas, Botella, Quero, Baños et al., 2014, y Cárdenas et al., 2013.

Entrevista estructurada ADIS-IV (Brown, DiNardo, y Barlow, 1994). Empleada para el diagnóstico de los trastornos de ansiedad siguiendo los criterios del DSM-IV. Evalúa los criterios diagnósticos y el grado de temor y de evitación que presenta la persona ante una serie de situaciones sociales, la frecuencia con la que se encuentra en dichas situaciones y si la ansiedad ante situaciones sociales es inmediata o anticipada. El instrumento indaga los episodios actuales y previos de ansiedad social, la etiología, la interferencia con el funcionamiento cotidiano y la posible presencia de ataques de pánico. La gravedad como la interferencia se miden en una escala Likert de 5 puntos en la que 0 es "nada grave" y 4 es "muy grave".

Inventario de Ansiedad Rasgo-Estado (IDA$R E$; Spielberger, y Díaz-Guerrero, 1975). Versión adaptada a población mexicana en español del STAI (State Trait Anxiety Inventory). Son dos escalas separadas de auto-evaluación que miden dos dimensiones de la ansiedad: la ansiedad rasgo (A-Rasgo) y la ansiedad estado (A-Estado). La escala A-rasgo consta de 20 afirmaciones en las que se pide a los participantes que describan cómo se sienten generalmente $(\alpha=0.86)$ y la escala A-estado, consta de 20 afirmaciones que requieren que los participantes indiquen cómo se sienten en un momento dado $(\alpha=0.54)$.

Inventario de Depresión de Beck (BDI; Beck Depression Inventory, Beck, 1961). Estandarizado en México por Jurado et al. (1998), $\operatorname{con} \alpha=0.94$. Consta de 2 factores constituidos por un total de 21 reactivos. El factor de síntomas afectivos-cognoscitivos está compuesto por 14 reactivos referentes a: tristeza, pesimismo, sensación de fracaso, insatisfacción, culpa, expectativas de castigo, auto-desprecio, auto-acusación, idea suicidas, episodios de llanto, irritabilidad, retirada social, indecisión y cambios en la imagen corporal. Por su parte, el factor de síntomas vegetativos y somáticos se encuentra conformado por 7 reactivos referentes a: lentitud, insomnio, fatiga, pérdida de apetito, pérdida de peso, preocupaciones somáticas y bajo nivel de energía. El inventario maneja una escala tipo likert de 0 a 3 donde 0 indica que no hay síntoma depresivo, 1 síntoma leve, 2 síntoma moderado y 3 síntoma grave. El punto de corte para el diagnóstico es de 14 o más.

Escala de miedo y evitación (adaptación de Marks y Mathews, 1979). En este cuestionario, el paciente y el terapeuta establecen cuatro situaciones o conductas que el paciente evita debido al pánico a la agorafobia. Califica su grado de evitación y miedo en una escala del 0 -10 donde $0=$ nunca lo evito y $10=$ siempre lo evito.

Opinión sobre el tratamiento (Borkovec y Nau, 1972; adaptado por Botella et al., 2009). Cuestionario de cuatro preguntas que informan del nivel de satisfacción con el tratamiento, sí recomendaría el tratamiento a un amigo o familiar, sí considera útil el tratamiento para su caso y si cree que el tratamiento fue difícil de manejar o aversivo. En una escala de 1 (nada) a 10 (bastante).

Cuestionario de Presencia y Juicio de Realidad (Baños, Botella, García-Palacios, Villa, Perpiñá y Alcañiz, 2000; Baños, Quero, Salvador y Botella, 2005). Es un cuestionario de 33 ítems diseñado para medir distintos componen- 
tes del sentido de presencia en un entorno virtual. Contiene siete factores: implicación emocional, juicio de realidad y presencia, interacción y correspondencia externa, influencia de claridad del software, facilidad de software, satisfacción con la experiencia y atención.

\section{Equipo}

El hardware utilizado fue una computadora Pentium III (1000 KHZ, 256 MB de memoria, CD-ROM drive y tarjeta gráfica AGP, $64 \mathrm{MB}$ con soporte para Open GL), iWear ${ }^{\circledR}$, VR920, Vuzix, Rochester, NY, EUA; pantallas LCD con resolución de $640 \times 480,920.000$ píxeles, 32 grados de campo de vista y construido en 3 grados de libertad del head-tracker.

\section{Procedimiento}

Previo a la implementación de los programas de tratamiento se realizó la adaptación de los 3 sistemas transferidos. Se logró la transferencia tecnológica del grupo de investigación de la Universidad Jaime I. La transferencia consistió en dos sistemas de tratamiento basados en tecnologías de realidad virtual conformados por diversos escenarios tridimensionales para la evaluación y tratamiento de miedo a volar y agorafobia con/sin ataques de pánico, y un programa telepsicología para el tratamiento de miedo a hablar en público "Háblame".

Se realizó la adaptación y pertinencia a población mexicana de los ambientes de realidad virtual y de los instrumentos de evaluación empleados para el tratamiento de los trastornos de miedo a volar $(\mathrm{MaV})$, miedo a hablar en público (MHP) y agorafobia (Agora) que se habían utilizado en población española. El proceso de adaptación consistió en realizar los ajustes con acento mexicano a los audios en los escenarios virtuales, considerando el contexto social y cultural a la población mexicana, con la finalidad de lograr un mejor encuadre cultural, y la posibilidad de que el usuario tuviera mayor interacción y ejecución en los ambientes virtuales.
Se entrenó a los psicólogos terapeutas en la aplicación de los programas de intervención mediante realidad virtual y en el programa autoaplicado para miedo a hablar en público. Como parte de la transferencia tecnológica se recibió capacitación por el equipo de investigación del Laboratorio de Psicología y Tecnología (LabPsiTec) de la Universidad Jaime I.

Se ofreció el tratamiento psicológico con incorporación tecnológica en el Centro de Servicios Psicológicos de la Facultad de Psicología de la UNAM. En todos los casos se llevó a cabo la evaluación inicial a las personas que solicitaron el servicio psicológico, a través del cual se exploraba si se cumplían los criterios de inclusión para cualquiera de los 3 estudios. Se realizaron 2 sesiones de evaluación por el terapeuta y una sesión de evaluación independiente por otro terapeuta con la finalidad de validar el diagnóstico clínico. Se aplicaron los siguientes instrumentos de evaluación y autoinformes. Una vez establecido el diagnóstico, se informó a los participantes en qué consistía el estudio y se les pidió que firmaran el informe de consentimiento. Las personas que aceptaron participar en el estudio correspondiente de acuerdo a su motivo de consulta.

\section{Tratamientos}

Programa de telepsicología para el tratamiento de miedo a hablar en público. El programa de telepsicología y el software Háblame (Botella et al., 2007) está integrado por tres módulos genéricos dedicados a la evaluación, el tratamiento y la prevención de recaídas. El módulo de psicoeducación, está dedicado a brindar a los participantes información sobre la naturaleza y las características de la ansiedad, el miedo y las fobias. La reestructuración cognitiva instruye a los participantes a identificar y confrontar pensamientos automáticos negativos en el MHP. En cuanto a la exposición, los usuarios se exponen a situaciones de hablar en público mediante vídeos de audiencias reales. Al final del tratamiento el programa Háblame refuerza a los participantes por su esfuerzo y les invita a continuar exponiéndose hasta superar todas las situaciones temidas. 
Programa de tratamiento para miedo a volar en avión mediante realidad virtual. El programa Virtual Flight desarrollado por Botella et al. (2001) incluyó tres modelos gráficos tridimensionales que cuentan con elementos que permiten tener altos índices de presencia al contar con diferentes estímulos para incrementar la dificultad de la situación temida, como: género del usuario, condiciones climáticas cambiantes, etc. El programa incluye 3 escenarios de realidad virtual: (1) La habitación: Es un escenario diseñado para trabajar con ansiedad anticipatoria; (2) El aeropuerto: En este escenario podemos simular las horas y los minutos previos al vuelo; (3) El avión: Aquí el paciente está sentado en el avión cerca de la ventana con una persona a su lado. Entonces, el proceso del vuelo comienza con la siguiente secuencia: (1) se proyectan las medidas de seguridad en una pantalla enfrente del usuario, (2) el capitán da la bienvenida a los pasajeros y reporta la información sobre la altura y las condiciones climáticas durante el vuelo, (3) despegue: después de las instrucciones de abrocharse los cinturones, el sonido de los motores es más fuerte y el avión empieza a encarrerarse; el avión acelera y despega, (4) vuelo: durante el vuelo podemos cambiar las condiciones climáticas, generar turbulencia, rayos, depresiones, y (5) aterrizaje: el capitán informa a los pasajeros sobre la proximidad del aterrizaje y les pide que se abrochen los cinturones. Después, el avión inicia el procedimiento de aterrizaje.

Programa de tratamiento para Agorafobia mediante realidad virtual. Se emplearon seis ambientes virtuales diseñados y evaluados por el grupo de investigación de Botella et al., (2004), para el tratamiento del trastorno de pánico con agorafobia: la sala de un departamento, el metro, autobús, un centro comercial y un túnel. En cada escenario, la exposición a los estímulos interoceptivos y externos puede llevarse a cabo de forma simultánea. Las sensaciones físicas que pueden ser simuladas son (1) palpitaciones y dificultad para respirar, con tres niveles de intensidad (leve, moderado y acelerado), y (2) efectos visuales (visión de túnel, visión borrosa y visión doble). Además, cada acción en el mundo virtual puede repetirse tanto veces como sea necesario para cada paciente.

\section{RESULTADOS}

\section{Equivalencia de los grupos}

Con la finalidad de determinar que existía homogeneidad en los tres grupos de tratamiento (Agora, MaV y MHP) en variables demográficas y de diagnóstico, se realizó un análisis estadístico antes de la intervención.

Con relación a las variables categóricas se computó la Chi cuadrada $\left(\chi^{2}\right)$. No se observaron diferencias significativas en las variables como sexo $\left(\chi^{2}=1.85\right.$, n.s. $)$, nivel de estudios $\left(\chi^{2}=\right.$ 0.33 , n.s. $)$ y diagnóstico $\left(\chi^{2}=0.00\right)$. Se calculó la prueba de U de Mann Whitney para la variable edad ( $z=-0.840$, n.s. $)$ donde no se mostraron diferencias significativas entre los grupos.

Para las variables continuas tampoco se encontraron diferencias significativas con respecto a las medidas clínicas grado de temor $(z=$ -1.67 , n.s.), grado de evitación ( $z=-1.88$, n.s.), grado de creencia ( $z=-0.23$, n.s.), ansiedad estado $(z=-0.50$, n.s. $)$ y depresión $(z=-1.59$, n.s.).

\section{Análisis Inter-grupales}

Se efectuó un análisis Kruskal-Wallis para determinar si existían diferencias entre las puntuaciones en las medidas clínicas de los tres diferentes grupos de tratamiento: Agorafobia, miedo a volar y miedo a hablar en público. Las distribuciones en los valores para las medidas de grado de temor, grado de evitación, grado de creencias, ansiedad estado y depresión fueron similares para todos los grupos, tal como se pudo analizar visualmente por medio de un diagrama de caja.

En valores de las medianas para las medidas clínicas existían diferencias estadísticamente significativas entre los grupos para el grado de temor $\left(\chi^{2}=6.54, p<.05\right)$, grado de creencia $\left(\chi^{2}=12.41\right.$, $p<.01)$ y ansiedad $\left(\chi^{2}=16.07, p<.001\right)$.

Para las medidas grado de evitación $\left(\chi^{2}=\right.$ 5.53 , n.s. $)$ y depresión $\left(\chi^{2}=1.57\right.$, n.s. $)$ no existieron diferencias estadísticamente significativas entre las medianas.

Para la medida de presencia y juicio de realidad $\left(\chi^{2}=3.99\right.$, n.s. $)$ tampoco existieron dife- 
rencias significativas entre los grupos lo cual indica que el nivel de presencia y juicio de realidad en los tres programas de tratamiento fue igual. En las medidas relacionadas con la satisfacción en el tratamiento no existieron diferencias estadísticamente significativas para las preguntas relacionadas con la satisfacción con el tratamiento $\left(\chi^{2}=4.27\right.$, n.s. $)$, recomendaría el tratamiento a un amigo $\left(\chi^{2}=3.20\right.$, n.s. $)$, el tratamiento podría ser útil para tratar otros problemas psicológicos $\left(\chi^{2}=5.48\right.$, n.s.) y si percibieron el tratamiento como aversivo $\left(\chi^{2}=1.17\right.$, n.s. $)$.

Encontramos diferencias estadísticamente significativas entre los grupos para la medida del grado de utilidad en el programa de tratamiento $\left(\chi^{2}=6.57, p<.05\right)$. Todas las comparaciones por pares fueron realizadas utilizando el procedimiento de Dunn (1964) con el ajuste de Bonferroni para comparaciones múltiples.

\section{Análisis Intra-grupales}

Se realizaron comparaciones entre las medidas pre- y post- de cada uno de los participantes de cada uno de los grupos de tratamiento (Agora, MaV y MHP) mediante la prueba no paramétrica Wilcoxon para muestras relacionadas; esta última es utilizada para medir cambios antes y después en grupos en los que cada sujeto funciona como su propio control.

En la Tabla 1 se muestran los resultados de los análisis intra-grupales para el programa de tratamiento de agorafobia. Se encontraron diferencias estadísticamente significativas entre el pre-test y el post-test para el grado de temor $(Z=-2.70, p<.01)$, grado de evitación $(Z=$ $-2.67, p<.01)$, y grado de creencia en el pensamiento negativo $(Z=-2.55, p<.05)$.

Para las medidas de ansiedad y depresión, se encontraron diferencias estadísticamente significativas en el caso de depresión $(Z=-2.31, p<$ $.05)$; sin embargo, no se encontraron diferencias significativas para ansiedad $(Z=-1.40$, n.s.).

Los resultados que se obtuvieron en el análisis intra-grupal de la aplicación del programa de tratamiento para miedo a volar en avión se encuentran en la Tabla 2. Tal y como se puede observar, se encontraron diferencias estadísticamente significativas entre el pre-test y el posttest para el grado de temor $(Z=-2.25, p<.05)$, grado de evitación $(Z=-1.90, p<.05)$, $\mathrm{y}$ grado de creencia en el pensamiento negativo $(Z=$ $-2,69, p<.01)$.

Tabla 1. Grado de cambio pretest - postest observado después del Programa de Tratamiento de Agorafobia (Prueba Wilcoxon; $N=9$ )

\begin{tabular}{lccrcc}
\hline \multirow{2}{*}{\multicolumn{1}{c}{ Variable }} & \multicolumn{2}{c}{ Pre } & \multicolumn{2}{c}{ Post } & $Z$ \\
\cline { 2 - 5 } & Media & $D T$ & Media & $D T$ & $-2.70^{* *}$ \\
\hline Grado de Temor & 8.77 & 1.64 & 4.22 & 2.04 & $-2.67^{* *}$ \\
Grado de Evitación & 8.55 & 1.66 & 2.22 & 2.86 & $-2.55^{*}$ \\
Grado de Creencia & 8.00 & 2.23 & 3.00 & 1.87 & -1.40 \\
Ansiedad Estado & 39.55 & 14.52 & 32.44 & 14.23 & $-2.03^{*}$ \\
Depresión & 15.00 & 7.93 & 6.88 & 4.98 & \\
\hline
\end{tabular}

$* p<.05, * * p<.01$.

Tabla 2. Grado de cambio pretest - postest observado después del Programa de Tratamiento de Miedo a volar en avión (Prueba Wilcoxon; $N=9$ )

\begin{tabular}{lccccc}
\hline \multirow{2}{*}{\multicolumn{1}{c}{ Variable }} & \multicolumn{2}{c}{ Pre } & \multicolumn{2}{c}{ Post } & $Z$ \\
\cline { 2 - 5 } & Media & $D T$ & Media & $D T$ & $-2.25^{*}$ \\
\hline Grado de Temor & 6.11 & 3.65 & 1.88 & 2.31 & $-1.90^{*}$ \\
Grado de Evitación & 5.00 & 4.06 & 1.55 & 1.58 & $-2.69^{* *}$ \\
Grado de Creencia & 7.55 & 2.65 & 1.22 & 0.83 & -1.78 \\
Ansiedad Estado & 37.44 & 8.01 & 33.88 & 7.76 & $-2.32^{*}$ \\
Depresión & 9.11 & 5.66 & 5.22 & 3.86 & \\
\hline
\end{tabular}

$* p<.05, * * p<.01$. 
Tabla 3. Grado de cambio pretest - postest observado después del Programa de Tratamiento de Miedo a Hablar en Público (Prueba Wilcoxon; $\mathrm{N}=9$ )

\begin{tabular}{lccccc}
\hline \multirow{2}{*}{ Variable } & \multicolumn{2}{c}{ Pre } & \multicolumn{2}{c}{ Post } & $Z$ \\
\cline { 2 - 5 } & Media & $D T$ & Media & $D T$ & $-2.71^{* *}$ \\
\hline Grado de Temor & 7.00 & 2.12 & 3.11 & 1.26 & $-2.69^{* *}$ \\
Grado de Evitación & 8.44 & 1.13 & 3.66 & 0.50 & $-2.72^{* *}$ \\
Grado de Creencia & 8.55 & 0.88 & 3.66 & 0.50 & $-2.67^{* *}$ \\
Ansiedad Estado & 28.33 & 10.39 & 8.88 & 3.55 & $-2.68^{* *}$ \\
Depresión & 11.33 & 4.35 & 4.22 & 1.98 &
\end{tabular}

Para las medidas de ansiedad y depresión, se encontraron diferencias estadísticamente significativas en depresión $(Z=-2,32, p<.05)$, pero no para ansiedad $(Z=-1,78$, n.s. $)$.

Se realizó análisis intra-grupal de las medidas pre- y post- del programa de tratamiento para el miedo a hablar en público. Tal como se puede observar en la Tabla 3, encontramos diferencias estadísticamente significativas entre el pre-test y el post-test para el grado de temor $(Z=-2,71, p<.01)$, grado de evitación $(Z=$ $-2,69, p<.01)$, y grado de creencia en el pensamiento negativo $(Z=-2,72, p<.01)$.

Para las medidas de ansiedad y depresión se encontraron diferencias estadísticamente significativas tanto para depresión $(Z=-2.68, p$ $<.01)$ como para la medida de ansiedad $(Z=$ $-2.67, p<.01)$.

\section{DISCUSIÓN}

El uso de las nuevas tecnologías en el ámbito de la psicología clínica ha dejado de ser una posibilidad, y hoy en día estas herramientas han probado ser eficaces y útiles para las distintas tareas que los profesionales realizan en este ámbito. Esto es el vertiginoso desarrollo de las tecnologías informáticas que ha permitido que sus aplicaciones en el campo de la salud sean cada vez más novedosas y flexibles. Así como la creciente práctica de someterlos a prueba, obtener evidencia empírica acerca de estos sistemas y ponerlos a disposición de un mayor sector de la población.

El propósito principal del presente trabajo fue la generalización y validación transcultural de tres intervenciones innovadoras en población mexicana para el tratamiento de tres desórdenes de ansiedad: (a) tratamiento para miedo a volar en avión mediante exposición por realidad virtual, (b) tratamiento para agorafobia $\mathrm{y} /$ pánico mediante exposición por realidad virtual; y c) programa de telepsicología para el tratamiento de miedo a hablar en público. Los resultados del presente estudio confirman la eficacia y efectividad de los tratamientos mediados por tecnologías avanzadas, validados en estudios previos realizados en población española (Botella et al., 2011; Botella et al., 2007; Botella et al., 2004), poniendo de manifiesto la reducción de sintomatología fóbica, ansiedad, evitación grado de creencia en el pensamiento negativo, así como variables de inmersión y juicio de presencia en una muestra clínica mexicana.

Los hallazgos apoyan la hipótesis declarada por Good y Kleinman (1985), acerca de la conveniencia de la validación transcultural de los protocolos de tratamiento, teniendo en cuenta que la percepción emocional de los problemas, así como las expresiones idiomáticas tienen que ser adaptados al contexto cultural y social de la población objetivo.

Por otra parte, en cuanto a la eficacia de los tratamientos, los resultados que se presentan ofrecen datos al comparar una evaluación antes y después de recibir el tratamiento. Se encontraron cambios estadísticamente significativos en todos los indicadores clínicos de fobia específica a volar en avión, miedo a hablar en público y agorafobia; así como de sintomatología asociada ansiosa/depresiva en los tres programas de tratamiento.

Los participantes mostraron reducción en los síntomas de evitación, lo que implicaría una mayor habilidad del participante para generalizar lo aprendido durante la sesión de tratamiento mediado por tecnología de realidad virtual y 
telepsicología a situaciones de la vida cotidiana; es decir que entre más similar sea el entorno de aprendizaje a la realidad, mayor será la probabilidad de que lo adquirido en el ambiente virtual se transfiera a la situación real. Estos resultados confirman los hallazgos de Anderson, Jacobs, y Rothbaum (2004), Botella et al., (2009) y Wiederhold y Wiederhold (2006) acerca de que el empleo de la realidad virtual, favorece el control, seguridad y confidencialidad al presentar al paciente los estímulos temidos y elementos significativos asociados al trastorno, lo que contribuye a reducir la evitación, por tanto, potenciar la generalización de cambio en la exposición a estímulos reales. De esta forma el uso de la técnica de exposición por realidad virtual facilita el entrenamiento y el aprendizaje de nuevos reportorios conductuales, puesto que el participante no tiene que esperar a que los sucesos ocurran en la vida real sino que se pueden producir y reproducir durante la sesión de tratamiento (De la Rosa y Cárdenas, 2013).

Con respecto a la satisfacción de los participantes al concluir el tratamiento, no se encontraron diferencias de preferencia entre el programa auto-aplicado y los tratamientos mediante realidad virtual, lo cual apoya la hipótesis de que los participantes consideraron que los tratamientos con incorporación tecnológica son lógicos y útiles para abordar la problemática emocional, lo que está en concordancia con la línea de varios autores (García-Palacios, Hoffman, y Fabregat, 2007; Rizzo, Gerardi, Rothbaum, Ressler, y Heekin, 2008; Titov, Dear, Johnson, y Terides, 2012) al manifestar que a los participantes les es más fácil dar el primer paso para enfrentar su miedo cuando pueden hacerlo a través de ambientes virtuales.

Por lo tanto, con base en lo establecido en Eje 2, sobre efectividad o utilidad clínica de la guía para los tratamientos empíricamente validados (APA, 1995), que contempla el grado en que los pacientes aceptan el tratamiento; podemos decir que la incorporación de las tecnologías de realidad virtual y/o telepsicología para el tratamiento de fobias puede ofrecer mejoras en los tratamientos con evidencia empírica ya existente.

Los resultados obtenidos muestran beneficios a los usuarios en dos niveles: (a) a los pacientes que presentaron sintomatología fóbica, ya que se contaron con programas de tratamiento que han probado su eficacia, y (b) para el terapeuta, debido a que el empleo de tecnología de realidad virtual representa varias ventajas para el clínico; resulta menos costosa e igual de eficaz que la exposición en vivo, con la diferencia que en exposición mediante realidad virtual es posible tener mejor control de los estímulos, lo que no se puede controlar en una exposición en vivo; además es menos costoso exponer al paciente desde el consultorio o internet a las situaciones temidas que hacerlo en situaciones reales. El paciente tiene mayor confidencialidad al exponerse a situaciones y mayor seguridad, ya que el sistema de realidad virtual brinda un ambiente controlado y protegido que permite al usuario explorar, experimentar y actuar en situaciones que siempre ha considerado amenazadoras (Cárdenas et al., 2013).

En este sentido, se destaca la validación transcultural de los programas de tratamiento con incorporación tecnológica, que contribuye para optimizar tratamientos y sistemas de valoración psicológica que beneficiarán los servicios de salud mental, ya que la evidencia nos dicta que los avances tecnológicos incorporados al tratamiento psicológico, representan herramientas fundamentales para el otorgamientos de servicios de salud del siglo XXI.

Los resultados derivados de los estudios permitirán abrir nuevas líneas de investigación de una variedad de trastornos psicológicos, además de evaluar la efectividad de este tipo de herramientas tecnológicas como apoyo en el tratamiento psicológico. Esperamos que los datos empíricos de estos estudios contribuyan a la toma de decisiones de los profesionales para integrar la mejor evidencia de investigación disponible a su experiencia práctica y a los atributos de valores, preferencias y circunstancias de sus pacientes. Lo cual implica que los profesionales tratarán de maximizar la probabilidad de que sus clientes reciban la intervención más eficaz.

\section{REFERENCIAS}

American Psychological Association (1995). Task force on psychological intervention guidelines. Template for 
developing guidelines: Interventions for mental disorders and psychological aspects of physical disorder. Washington, D.C: APA.

Anderson, P., Jacobs, C., \& Rothbaum, B.O. (2004). Computer-supported cognitive behavioral treatment of anxiety disorders. Journal of Clinical Psychology, 60, 253-267.

Beck, A.T., Ward, CH., Mendelson, M., Mock, J., \& Erbaugh, J. (1961). An inventory for measuring depression. Archives of General Psychiatry, 4, 561-571.

Borkovec, T.D., \& Nau, S.D. (1972). Credibility of analogue therapy rationales. Journal of Behaviour. Therapy and Experimental Psychiatry, 3, 257-260.

Botella, C. (2011). Tratamientos Psicológicos y salud mental. Publicaciones Universidad Jaime I, D. L.

Botella, C. Osma, J., García-Palacios, A., Quero, S., \& Baños, R. (2004). Treatment of flying phobia using virtual reality: data from a year follow-up using a multiple baseline design. Clinical Psychology \& Psychotherapy, 11, 311-323

Botella, C., Baños, R.M., García-Palacios, A., Quero, S., Guerrero, B., Liaño, V., \& Perpiñá, C. (2005). Using traditional strategies in a virtual World for the treatment of pathological grief. Proceedings at 5th International Congress of Cognitive Psychotherapy (ICCP 2005), Göteborg, Sweden.

Botella, C., Gallego, M.J., García-Palacios, A., Guillen, V., Baños, R.M., Quero, S., \& Alcañiz, M. (2011). An Internet-Based Self-Help Treatment for Fear of Public Speaking: A Controlled Trial. Cyberpsychology, Behavior and Social Network, 13, 1-15.

Botella, C., García-Palacios, A., Villa, H., Baños, R.M., Quero, S., Alcañiz, M., \& Riva, G. (2007). Virtual Reality Exposure in the Treatment of Panic Disorder and Agoraphobia: A Controlled Study. Journal of Clinical Psychology and Psychotherapy, 14, 164-175.

Botella, C., Quero, S., Serrano, B., Baños, R., y GarcíaPalacios, A. (2009). Avances en los tratamientos psicológicos: la utilización de las nuevas tecnologías de la información y la comunicación. Anuario de Psicología, 40, 155-170.

Brown, T.A., DiNardo, P.A., \& Barlow, D.H. (1994). Anxiety Disorders Interview Schedule for DSM-IV (ADIS-IV)-Adults version. Albany, NY: Graywind.

Cárdenas, G., \& De la Rosa, A. (2012). Using Virtual Reality for PTSD Treatment in Criminal Violence Victims. Journal of CyberTherapy \& Rehabilitation, 5, 65-67.

Cárdenas, G., Botella, C., Quero, S., De la Rosa, A., y Baños, R. (2012, en prensa). Programa de Telepsicología para el Tratamiento de la Fobia a hablar en público en Población Mexicana. Revista Psicología Iberoamericana.

Cárdenas, G., De la Rosa, A., Durán, X., \& Bouchard, S. (2014, in press). PTSD treatment using virtual reality for victims of criminal and urban violence. In P. Cipress, \& S. Serino (Eds.), Virtual Reality Technologies, Medical Applications and Challenges. NOVA Publisher.

Cárdenas, G., De la Rosa, A., Flores, L., \& Durán, X. (2013, in press). A Controlled trial for PTSD in Mexican victims of criminal violence. Proc. IEEE Intl. Conf.

De la Rosa, A., y Cárdenas, G. (2012). Trastorno por estrés postraumático: eficacia de un programa de tratamiento mediante realidad virtual para víctimas de violencia criminal en población mexicana. Anuario de psicología, 42, 377-391.

Difede, J., Cukor, J., Jayasinghe, N., Patt, I., Jedel, S., Spielman, L., et al. (2007). Virtual reality exposure therapy for the treatment of posttraumatic stress disorder following September 11, 2001. Journal of Clinical Psychiatry, 68, 1639-1647.

Dunn, O.J. (1964). Multiple comparisons using rank sums. Technometrics, 6, 241-252.

Gallego, M., Botella, C., García, A., Quero, S., y Baños, R. (2002). Validación de la escala de autoverbalizaciones durante la situación de hablar en Público (SSPS). CIBER Fisiopatología Obesidad y Nutrición (CB06/03), Instituto de Salud Carlos III, España.

García-Palacios, A., Botella, C., Hoffman, H., \& Fabregat, S. (2007). Comparing acceptance and refusal rates of virtual exposure versus in vivo exposure by patients with specific phobia. CyberPsyhology and Behaviour, 10, $722-724$.

Good, B.J., \& Kleinman, A. M (1985). Culture and anxiety: Cross cultural evidence for the patterning of anxiety disorders. In A.H. Tuma \& J.D. Master (Eds), Anxiety and the anxiety disorders (pp. 297-323). Hillsdale, NJ: Erlbaum.

Holden, M.K. (2005). Virtual Environments for Motor Rehabilitation: Review. CyberPsychology and Behaviour, 8,187-211.

Jurado, S., Villegas, M., Méndez, L., Rodríguez, F., Loperena, V., y Varela, R. (1998). La estandarización del Inventario de Depresión de Beck para los residentes de la ciudad de México. Salud Mental, 21, 26-31.

López-Soler, C., Castro, M., Alcántara, M., y Botella, C. (2011). El sistema de realidad virtual EMMA-infancia en el tratamiento psicológico de un menor con estrés postraumático. Revista de Psicopatología y Psicología Clínica, 16, 189-206.

Marks, I.M., \& Mathews, A.M. (1979). Brief standard self-rating scale for phobic patients. Behaviour Research and Therapy, 17, 263-267.

Medina, M., Borges, G., Lara, C., Benjet, C., Blanco, J., Fleiz, C., Villatoro, J., Rojas, E., Zambrano, J., Casanova, L., y Aguilar, S. (2003). Resultados de la Encuesta Nacional de Epidemiología Psiquiátrica en México. Salud Mental, 26, 1-16. 
Merians, A.S., Fluet, G.G., Qiu, Q., Saleh, S., Lafond, I., \& Adamovich, S.V. (2010). Integrated arm and hand training using adaptive robotics and virtual reality simulations. Proceedings of the 2010 International Conference on Disability, Virtual Reality and Associated Technology, 213-222.

Moriana, J. A., y Martínez, V. A. (2011). La psicología basada en la evidencia y el diseño y evaluación de tratamientos psicológicos eficaces. Revista de Psicopatología y Psicología Clínica, 16, 81-100.

Parsons, T.D., \& Rizzo, A.A. (2008). Affective Outcomes of Virtual Reality Exposure Therapy for Anxiety and Specific Phobias: A Meta-Analysis. Journal of Behavior Therapy and Experimental Psychiatry, 39, 250-261.

Powers, M., \& Emmelkamp, P.M.G. (2008). Virtual reality exposure therapy for anxiety disorders: A metaanalysis. Journal of Anxiety Disorders, 22, 561-569.

Riva, G. (2011). The Key to Unlocking the Virtual Body: Virtual Reality in the Treatment of Obesity and Eating Disorders. Journal of Diabetes Science and Technology, 5, 283-292.

Rizzo, A. (2010). Virtual Iraq/Afghanistan and how it is helping some troops and vets with PTSD. Available at Veterans Today: http://www.veteranstoday. com/2010/07/29/virtual-iraqafghanistan-and-how-itis-helping-some-troops-and-vets-with-ptsd/

Rizzo, A., Difede, J., Rothbaum, B.O., \& Reger, G. (2010). Virtual Iraq/Afghanistan: development and early evaluation of a virtual reality exposure therapy system for combat-related PTSD. Annals of the New York Academy of Sciences (NYAS), 12, 114-125.

Rizzo, A., Gerardi, M., Rothbaum, B.O., Ressler, K., \& Heekin, M. (2008). Virtual reality exposure therapy using a virtual Iraq: Case report. Journal of Traumatic Stress, 21, 209-213.
Rizzo, A., Parsons, T.D., Lange, B., Kenny, P., Buckwalter, J.G., Rothbaum, B.O., Difede, J., Frazier, J., Newman, B., Williams, J., \& Reger, G. (2011). Virtual Reality Goes to War: A Brief Review of the Future of Military Behavioral Healthcare. Journal of Clinical Psychology in Medical Settings.

Rizzo, A., Buckwalter, J.G., Forbell, E. Difede, J., Rothbaum, B.O., Lange, B., Koenig, S., \& Talbot, B. (2013). Virtual Reality Applications to Address the Wounds of War. Psychiatric Annals, 43,123-138.

Rizzo, A., Lange, B., \& Koenig, S. (2014). Clinical Virtual Reality. In K.S. Hale, \& K.M. Stanney (Eds.), Handbook of Virtual Environments: Design, Implementation, and Applications. CRC Press.

Rothbaum, B.O., Hodges, L., Ready, D., Graap, K., \& Alarcon, R. (2001). Virtual reality exposure therapy for Vietnam veterans with posttraumatic stress disorder. Journal of Clinical Psychiatry, 62, 617-622.

Rubin, A. (2008). Practitioners Guide to Using Research for Evidence-Based Practice. New Jersey: John Wiley \& Sons, Inc.

Sackett, D.L, Straus, S., Richardson, S., Rosenberg, W., \& Haynes, R.B. (2000). Evidence-based medicine: how to practice and teach EBM. $2^{\text {nd }}$. ed. London, U.K.: Churchill Livingstone.

Titov, N., Dear, B. F., Johnson, L., \& Terides, M. (2012). Transdiagnostic internet treatment for auxiety and depression. Revista de Psicopatología y Psicología Clínica, 17, 237-260.

Wiederhold, B.K., \& Wiederhold, M.D. (2006). The future of cybertherapy: Improved options with advanced technologies. En G. Riva, C. Botella, P. Légeron, \& G. Optale (Eds.), Internet and Virtual Reality as Assessment and Rehabilitation Tools for Clinical Psychology \& Neuroscience. Amsterdam: IOS Press. 